\title{
Correction: Rating Hospital Performance in China: Review of Publicly Available Measures and Development of a Ranking System
}

Shengjie Dong ${ }^{1 *}, \mathrm{MPH}$; Ross Millar ${ }^{2 *}, \mathrm{PhD} ;$ Chenshu Shi $^{3}, \mathrm{MSc}$; Minye Dong ${ }^{1}, \mathrm{MPH}$; Yuyin Xiao ${ }^{1}, \mathrm{MPH} ; \mathrm{Jie} \mathrm{Shen}^{4}$, $\mathrm{MD}$; Guohong $\mathrm{Li}^{1,4}, \mathrm{PhD}$

\footnotetext{
${ }^{1}$ School of Public Health, Shanghai Jiao Tong University School of Medicine, Shanghai, China

${ }^{2}$ Health Services Management Centre, University of Birmingham, Birmingham, United Kingdom

${ }^{3}$ Center for Health Technology Assessment, China Hospital Development Institute, Shanghai Jiao Tong University, Shanghai, China

${ }^{4}$ China Hospital Development Institute, Shanghai Jiao Tong University School of Medicine, Shanghai, China

*these authors contributed equally
}

\section{Corresponding Author:}

Guohong $\mathrm{Li}, \mathrm{PhD}$

China Hospital Development Institute

Shanghai Jiao Tong University School of Medicine

227 South Chong Qing Road

Shanghai, 200025

China

Phone: 862163846590

Email: guohongli@sjtu.edu.cn

\section{Related Article:}

Correction of: https://www.jmir.org/2021/6/e17095

(J Med Internet Res 2021;23(6):e31370) doi: 10.2196/31370

In "Rating Hospital Performance in China: Review of Publicly Available Measures and Development of a Ranking System" (J Med Internet Res 2021;23(6):e17095) the authors noted one error.

An author was incorrectly included in the authorship list on the published paper. This author has been removed from the corrected version of the manuscript. The correct author list is
Shengjie Dong, Ross Millar, Chenshu Shi, Minye Dong, Yuyin Xiao, Jie Shen, Guohong Li.

The correction will appear in the online version of the paper on the JMIR Publications website on June 22, 2021, together with the publication of this correction notice. Because this was made after submission to PubMed, PubMed Central, and other full-text repositories, the corrected article has also been resubmitted to those repositories.

This is a non-peer-reviewed article. Submitted 18.06.21; accepted 18.06.21; published 22.06.21.

Please cite as:

Dong S, Millar R, Shi C, Dong M, Xiao Y, Shen J, Li G

Correction: Rating Hospital Performance in China: Review of Publicly Available Measures and Development of a Ranking System

J Med Internet Res 2021;23(6):e31370

URL: https://www.jmir.org/2021/6/e31370/

doi: $10.2196 / 31370$

PMID:

(C) Shengjie Dong, Ross Millar, Chenshu Shi, Minye Dong, Yuyin Xiao, Jie Shen, Guohong Li. Originally published in the Journal of Medical Internet Research (https://www.jmir.org), 22.06.2021. This is an open-access article distributed under the terms of the Creative Commons Attribution License (https://creativecommons.org/licenses/by/4.0/), which permits unrestricted use, distribution, and reproduction in any medium, provided the original work, first published in the Journal of Medical Internet 
Research, is properly cited. The complete bibliographic information, a link to the original publication on https://www.jmir.org/, as well as this copyright and license information must be included. 